\title{
Retraction
}

\section{Retracted: Managing DSRC and WAVE Standards Operations in a V2V Scenario}

\section{International Journal of Vehicular Technology}

Received 13 March 2016; Accepted 13 March 2016

Copyright (C) 2016 International Journal of Vehicular Technology. This is an open access article distributed under the Creative Commons Attribution License, which permits unrestricted use, distribution, and reproduction in any medium, provided the original work is properly cited.

The article titled "Managing DSRC and WAVE Standards Operations in a V2V Scenario" [1] has been retracted as it was found to be essentially identical in content with the following published article: "Notes on DSRC and WAVE standards suite, its architecture, design, and characteristics" by Y. L. Morgan in IEEE Communications Surveys \& Tutorials.

\section{References}

[1] Y. L. Morgan, "Managing DSRC and WAVE standards operations in a V2V scenario," International Journal of Vehicular Technology, vol. 2010, Article ID 797405, 18 pages, 2010. 


\title{
Managing DSRC and WAVE Standards Operations in
} a V2V Scenario

\author{
Yasser L. Morgan \\ Department of Software Systems Engineering, University of Regina, 3737 Wascana Parkway Regina, Canada S4S 0A2 \\ Correspondence should be addressed to Yasser L. Morgan, yasser.morgan@uregina.ca
}

Received 12 December 2009; Revised 26 April 2010; Accepted 27 April 2010

Academic Editor: Shinsuke Hara

Copyright $\odot 2010$ Yasser L. Morgan. This is an open access article distributed under the Creative Commons Attribution License, which permits unrestricted use, distribution, and reproduction in any medium, provided the original work is properly cited.

The Dedicated Short-Range Communications (DSRC) standards suite is based on multiple cooperating standards mainly developed by the IEEE. In particular, we focus this paper on the core design aspects of DSRC which is called Wireless Access in Vehicular Environment (WAVE). WAVE is highlighted in IEEE 1609.1/.2/.3/.4. The DSRC and WAVE standards have been the center of major attention in both research and industrial communities. In 2008, WAVE standard was the third best seller standards in the history of the IEEE. This attention reflects the potential of WAVE to facilitate much of the vehicular safety applications. In this paper we present a fairly detailed tutorial of the WAVE standards. We extend the paper by describing some of the lessons learned from particular design approaches. We direct the reader to the landmark research papers in relevant topics. We alert the reader about major open research issues that might lead to future contribution to the WAVE design.

\section{DSRC Introduction and Historic Notes}

About one million traffic accidents occur annually in the USA. In 2003 alone, these accidents accounted for $\$ 230$ billion in damaged property, 2,889,000 nonfatal injuries, and 42,643 deaths. Evidently, most of those accidents are preventable by implementing comprehensive wireless communication mechanism to exchange vital safety and emergency information between moving vehicles [1]. Dedicated Short-Range Communications (DSRC) is a suite of standards at the heart of the communication of vehicular safety messages. The fast exchanging of safety messages, combined with knowledge about other moving vehicles that may not be visible to drivers in a timely manner extend the safety concepts beyond the dreams of most of the public [2].

Wireless Access in Vehicular Environment (WAVE) is a term used to describe the suite of IEEE P1609.x standards that are focused on MAC and network layers. WAVE is fairly complex and is built over the IEEE 802.11 standards by amending many tweaks to guarantee fast reliable exchange of safety messages. WAVE is the core part of DSRC; however, either of the two terms is commonly used arbitrarily. In some cases, the term DSRC is used as a more general term compared to WAVE.
The history leading to the development of current DSRC goes back almost a decade and a half. In the early 1990s, it became clear that road toll collection can be simplified by means of RFID transponders. Major industrial suppliers of electronic toll collection quickly discovered that further development on $915 \mathrm{MHz}$ might pave the road for much elegant breed of applications facilitating enhanced road safety and collision avoidance. The group of electronic toll suppliers along with other stake holders formed a consortium focused on DSRC development. Coincidently, multiple studies on vehicular safety and collision avoidance revealed that short-range communication ( $<100$ meters) would be sufficient for most safety application [3].

The DSRC community then attempted to standardize the $915 \mathrm{MHz}$ using the ASTM framework but quickly thought of the IEEE 802.11 approach and the $5.9 \mathrm{GHz}$ as a direct way to benefit from its ad hoc mode. The ad hoc mode of IEEE 802.11 resembles the situation of vehicle-to-vehicle communications and hence, simplifies the development of DSRC. Almost a decade of DSRC standards development has resulted in the IEEE 802.11p standards along with IEEE 1609.x, both standards represent together proposed DSRC suite of standards. 
DSRC is currently considered the most promising wireless standard that can be used to connect infrastructure (like roadside) to vehicle (I2V) and vehicle-to-vehicle (V2V). DSRC standard is based on the WiFi architecture. Relevant application layer consortiums like Vehicle-Infrastructure Integration (now called IntelliDrive), Cooperative Intersection Collision Avoidance Systems (CICAS) and others have developed their architect with DSRC services in mind [4].

The first task for the DSRC workgroup was basically to identify which wireless bands and technologies suits DSRC applications most. Table 1 simplifies the comparison. DSRS utilizes cheap, short-range, two-way Line-of-Sight (LoS) communications with sufficiently high bandwidth. DSRC applications lend themselves, naturally, to localized road communications which lead to a much desired distributed decentralized deployment. While the cellular or WiMax bandwidth may appear to provide higher bandwidth, sharing that bandwidth over geographically larger area brings down the effective bandwidth. The LoS constraint can also be mitigated by mounting DSRC devices at higher elevations which has been proven to work even in most crash scenarios. Further, DSRC causes limited latency compared to other candidate wireless technologies, but DSRC scores quit low when it comes to connectivity in mobile environment. There are two reasons for that, first, the short-range nature of the DSRC which leads to frequent change in the topology. We refer the reader to Morgan [5] where a full discussion into how to mitigate is included. Second is the fact limited number of DSRC devices exist right now. As the use of this system becomes more common, the coverage will improve and hence the continuous connectivity of mobile vehicles will be achieved.

It was also clear to the DSRC workgroup that adopting WiFi standards facilitates operations in infrastructure and ad hoc modes which map to I2V and V2V communications respectively. DSRC is not expected to replace other wireless technologies, nor is it expected to uniquely serve all vehicular communication needs, rather DSRC is seen as the main candidate for safety, short-range applications, subscription free services, road toll services, and other similar localized applications [6].

The US-FCC assigned the 5.850 to $5.925 \mathrm{GHz}$ band range as a freely licensed spectrum utilizing DSRC to enhance road safety. DSRC is capable of delivering $27 \mathrm{Mbps}$ data-rate by using a two way line-of-sight short-range radio which is significantly lower cost compared to cellular, WiMax or satellite communications as illustrated in Table 1. DSRC operates in stringent environment which requires; fast communications to maintain the connection with speeding vehicles at all times, strict QoS committed to predefined threshold delays for safety messages, minimal use of transmission power, and maintaining privacy and anonymity of roaming users in addition to many other environmental challenges.

The point of using minimal power is an important one. While the DSRC device is backed by large vehicular batteries, during our evaluation for the development of the standards we found that the higher use of signal power leads to other DSRC devices having to raise their signal power as well. The final outcome is a communication environment with so many interferences within its vicinity. In fact, in one of the testing we found the high power DSRC signal capable of hiding even the GPS signal which is typically a low power one. The key to successful reliable communication is lower signal power.

To assist the reader with the growing number of the acronyms in this industry, a glossary of used terms is added at the end of the article. As of this point, the paper is organized as follows. Section 2 introduces the reader to the necessary DSRC hardware. Section 3 defines the basic WAVE architecture and commonly used terms then identifies the WAVE reference model from a layered perspective.

Section 4 elaborates on core MAC services such as WAVE QoS, and Channel Coordination Function (CCF) of the 1609.4 with fairly extended discussion on synchronization tolerance issues. Section 4 defines WAVE communication modes. Section 5 visits the management of WAVE services in terms of persistence, service registration, discovery, and defining relevant management policies. Section 6 concludes and comments on future directions of DSRC. Each of the described subsections is followed by a discussion of the lessons learned, relevant design issues, and a sample scenario.

\section{DSRC Components}

In order to understand the WAVE architecture and various WAVE elements, it is important to learn about the typical DSRC devices and components. This section introduces DSRC components and uses simple example to elaborate possible DSRC street layout.

The DSRC network is built over two basic units; RoadSide Unit (RSU) and On-Board Unit (OBU). The RSU is, typically, a stationary unit that connects roaming vehicles to the access network, which, in turn, is connected to a larger infrastructure or to a core network. The OBU is, typically, a network device fixed in a roaming vehicle and is connected to both the DSRC wireless network and to an in-vehicle network. This simple architecture is illustrated in Figure 1.

The wireless connection between RSU and OBU is based on WAVE standards suite as illustrated in Figure 3. The cones shown in smaller dots in Figure 1 represent the RSU communication zones while the cone looking up represents the radio range of the OBU. As OBUs move between communication zones, vehicles exchange information with the roadside; in addition, vehicles use the same WAVE media to communicate with each other.

Figure 2 presents a plan view where the light posts shown off the green are equipped with RSUs. Each RSU has a communication zone indicated by the triangular cone and vehicles go through different communication zones as the drive on a highway. To define different WAVE communication zones, think of the term WAVE Basic Service Set (WBSS) as a unique identifier for each communication zone. Vehicles must associate with only one WBSS at a time. Hence, each communication zone has its own WBSS, in Figure 2 the first communication zone from the left is WBSS1 and the last is WBSS-4. Vehicles close to each other like Car$\mathrm{B}$ and Car-C may have a V2V communications like WBSS-5. 
TABLE 1: DSRC versus Other Wireless Technologies.

\begin{tabular}{|c|c|c|c|c|}
\hline & DSRC & FM Radio & WiMAX & Satellite \\
\hline Max Range (Km) & $<1$ & hundreds & $<10$ & thousands \\
\hline Data Rate mbps & $6-27$ & $>10 \mathrm{kbps}$ & $\begin{array}{c}<100 \mathrm{kbps} \\
3 \mathrm{G} 2-3 \mathrm{mbps}\end{array}$ & 100 \\
\hline Coverage & LoS-duplex & Area-1/2 duplex & Area-duplex & Area-duplex \\
\hline Cost (per bit) & $\approx$ zero & $\approx$ zero & $\$ \$$ & $\$ \$ \$$ \\
\hline Average Latency & Very Lo & $\mathrm{Hi}$ & Lo & Lo \\
\hline Mobile Connectivity & Lo & Lo & Very Hi & Very Hi \\
\hline
\end{tabular}

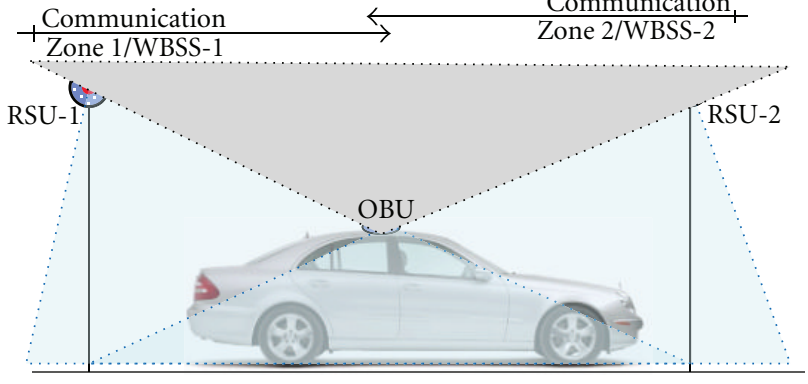

Figure 1: An OBU Roaming between two RSUs.

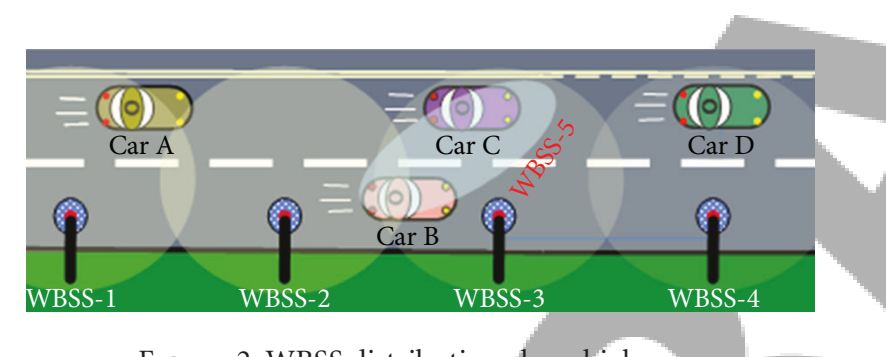

FIGURE 2: WBSS distribution along highways.

WBSS- 1 is an I2V and vehicles may be part of either I2 V V2V session at the same time.

The communication zone covered by each IEEE $802.11 \mathrm{p}$ RSU is limited to a maximum of $1 \mathrm{Km}$ diameter and uses $5.9 \mathrm{GHz}$ radio transmission. OBUs are expected to join the WBSS of the closest RSU, exchange information, and typically leave within limited time (mean estimate 3.6 seconds). The use of the term closest here is a reasonable approximation and the term is defined in more details Section 5.4. The limited lifetime of an OBU within specific WBSS communication zone imposes hard requirements on the design of the WAVE standards suite and on the nature of future DSRC applications as described in Section 4.2. DSRC networks use WAVE Short Messages Protocol (WSMP or WSM for short) to exchange safety information between vehicles and roadside or just between vehicles $[2,3]$.

WAVE devices employ an architecture that supports a predefined Control Channel $(\mathrm{CCH})$ and multiple Service Channels (SCHs). The CCH is used to transmit WSM and to announce WAVE services, while a carefully selected $\mathrm{SCH}$ is used for commercial application interactions and data transmissions.
This section defines WAVE basic architecture along with defining basic terms typically used in this domain. One might ask why we need so many standards. From a layered perspective, wireless communication between DSRC devices uses IEEE 802.11 and $802.11 \mathrm{p}$ standards as a lower layer close to the physical layer. The DSRC MAC layer uses IEEE WAVE standards which follows IEEE 1609.x standards. Then the higher layers employ IPv6 and other common protocol stacks like TCP/UDP for instance. This structure is illustrated in Figure 3.

A basic sample scenario that will be discussed in this section presents common vehicular communication either between vehicles or between vehicles and roadside. You can always upgrade different parts of the standards stacks as needed in the future without influencing other parts.

3.1. Current Focus. One of the biggest challenges when dealing with a suite of standards is the understanding of the functionalities of each standards and how multiple standards fit together to perform a homogeneous task. The term DSRC is typically used as an umbrella term that refers to the suite of standards shown in Figure 3.

Figure 3 illustrates the detailed functionalities of DSRC suite of standards where the arrows represent the standards' dependencies. Figure 3 is simplified to show the relations and dependencies but ignores tortuous details. For instance, since $802.11 \mathrm{p}$ depends ultimately on 802.11 , and since 802.11 uses CSMA/CA. Then you arrive to the conclusion that 802.11p follows the 802.11 CSMA/CA mechanisms. The DSRC standards suite relies on the CSMA/CA mechanism to mitigate the hidden/exposed node problems.

Instead of describing every detail related to DSRC and WAVE standards, this paper is centered on the IEEE 802.11p [7], IEEE 1609.3 [8] and IEEE 1609.4 [9] standards, and the reader may follow Figure 3 to discover details of the foundation technologies like 802.11. Also from Figure 3, it is clear that both 1609.1 [10] and 1609.2 [11] depend highly on 1609.3/.4 and on IEEE 802.11p. Therefore, the details of both $1609.1 / .2$ can only be clarified by relying on a description of 1609.3/.4. Since we expect no radical changes to 1609.3/.4, we focus this paper on WAVE MAC and network layer design that are fundamental, and necessary. We will be publishing another set of notes focused on 1609.1/.2 to provide complete view of WAVE standards. Most of the DSRC standards suite 


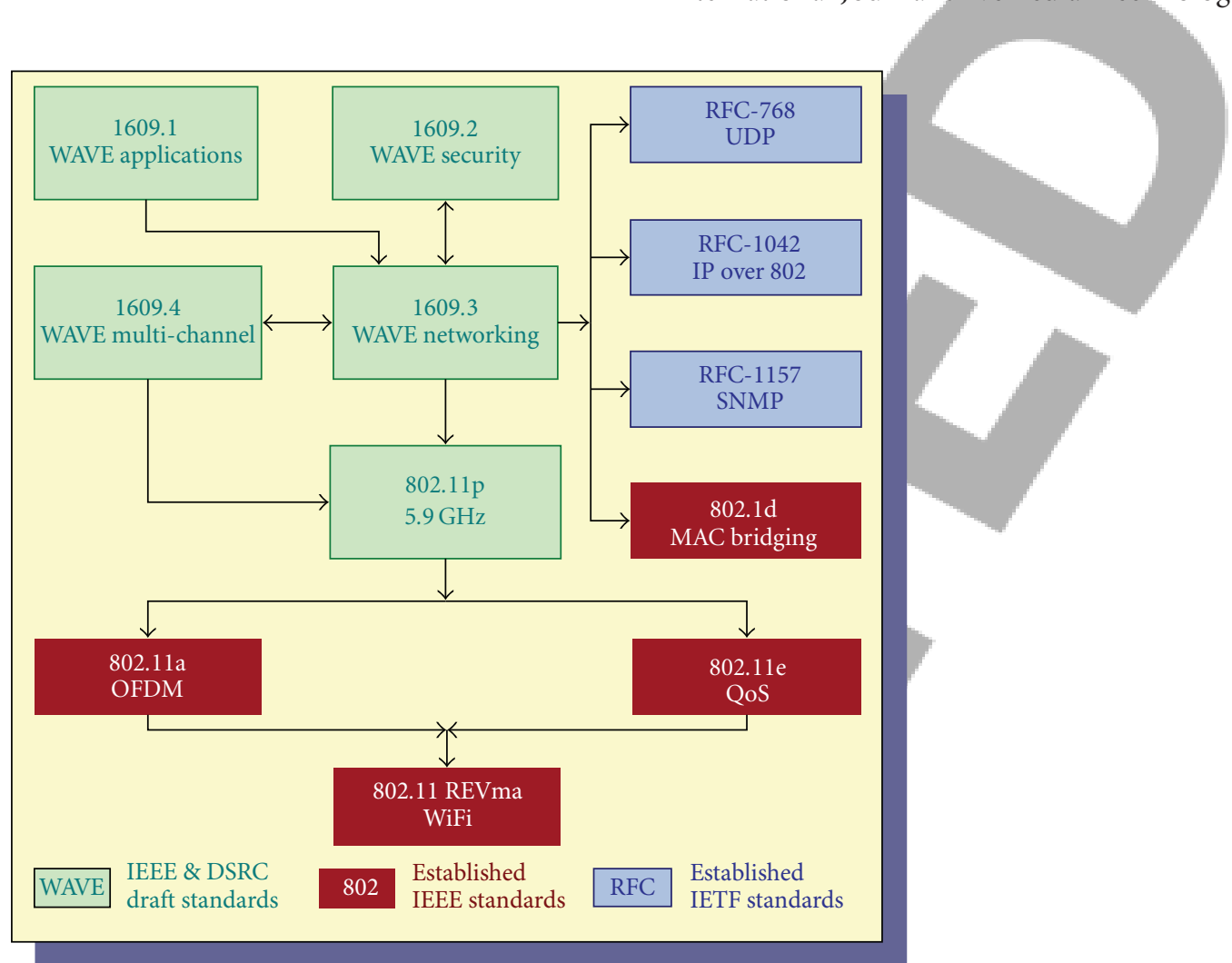

FIGURE 3: Simplified DSRC Standards Suite.

has been released as draft standards and the final RFCs could be released for the public by the time this paper is published.

3.2. Basics WAVE Radio Arrangement. The WAVE $5.9 \mathrm{GHz}$ spectrum was divided into smaller operational channels as shown in Figure 4. A typical WAVE device uses the Control Channel $\mathrm{CCH}$ and at least one Service Channel $\mathrm{SCH}$ as long as it remains connected to the same WBSS. The High Availability Low Latency (HALL) channel is being left for future use. In most current prototypes, channel 172 is unused. As a general rule, $\mathrm{CCH}$ (178) is exclusively used to communicate safety and control information while $\mathrm{SCH}$ is typically used to communicate IP-based services. Consequently, each communication zone must utilize channel 178 $(\mathrm{CCH})$ as a $\mathrm{CCH}$ used for safety messages, then, it may utilize one or more SCH of the available four service channels. Devices initiating WBSS are encouraged to avoid using the same SCHs selected by immediate neighbors.

WAVE relies on the IEEE 802.11a Orthogonal Frequency Division Multiplexing (OFDM) mechanism to provide data transmission rates of $9,12,18,24$, and $27 \mathrm{Mbps}$ for 0 $60 \mathrm{Km}$ /hour vehicle speed and 3, 4.5, 6, 9, and $12 \mathrm{Mbps}$ for $60-120 \mathrm{Km} /$ hour vehicle speed. The system comprises 52 sub-carriers, modulated using BPSK, QPSK, 16-QAM, or 64QAM. Convolution coding is used with a coding rate of $1 / 2$, $2 / 3$, or $3 / 4$. The data rates are determined by coding rate and modulation type $[12,13]$.

The DSRC divides channels into $10 \mathrm{Mhz}$ rather than the $22 \mathrm{Mhz}$ as designed in the IEEE 802.11. DSRC squeezes each channel bandwidth as indicated in the IEEE 802.11p [7], and then imposes a higher level management scheme. The scheme insures that the operating $\mathrm{SCH}$ in any vicinity is different from the operating SCH in any of the adjacent vicinities. This management scheme mitigates the potential for interference between channels.

3.3. The Use of Single and Multiple-Channel Radios. WAVE devices, such as RSU and OBU, are expected to be implemented using two types of radio devices. First is a singlechannel WAVE device which exchanges data and/or listens to only one RF channel at a time (commonly called single-channel device). Second is a multichannel WAVE device that exchanges data on one channel while, at least, actively listening on a second channel (commonly called multichannel device) [14].

In order to accommodate the limited capabilities of single-channel WAVE devices, yet allow interoperability with multichannel WAVE devices a synchronization mechanism is required to ensure that all WAVE devices monitor and/or utilize the Control Channel $(\mathrm{CCH})$ at common time intervals. Both $\mathrm{CCH}$ and Service Channel (SCH) intervals are uniquely defined with respect to an accurate common time reference.

The CCH/SCH design choices reflect the vehicular environment. The DSRC workgroup certainly learned lessons from the industry. Vehicles running on the road may utilize $\mathrm{CCH}$ and selected $\mathrm{SCH}$, while vehicles in a gas station within the same vicinity would select a different $\mathrm{SCH}$ to pay for gas and possibly download a map. Using different $\mathrm{SCH}$ 
preserves the emergency and safety communications on the critical $\mathrm{CCH}$. However, the design calls for accurate time synchronization [14].

Another advantage of the DSRC channel design is the availability of multiple service channels. The business case for adopting DSRC is simple. It is rather easy to attract businesses in urban areas to install DSRC devices especially since they are typically hungry for the bandwidth offered on service channels. Then, based on how the standards are created, safety messages will be carried for free over the control channel $\mathrm{CCH}$ as the devices switch from $\mathrm{SCH}$ to $\mathrm{CCH}$. In a sense, the core success factor of DSRC is embedded in its design. Therefore, urban areas could be covered by DSRC without active government contribution. For suburban and highways, a gradual deployment that favors areas of high accident rate seem to be the logical strategy.

The basic sample scenario here is the communications between two vehicles where one is equipped with a multichannel WAVE device and the other is equipped with a cheaper single-channel WAVE device. The objective is to allow the two different devices to switch to different service channels, yet, meet on the $\mathrm{CCH}$ during the scheduled time. Obviously, this requires careful synchronization as we describe in the next subsection.

3.4. Synchronization of WAVE Devices. WAVE devices use simple approach to synchronize. A typical WAVE device may visit the $\mathrm{CCH}$ for a period called $\mathrm{CCH}$ Interval $(\mathrm{CCHI})$ and is shown by the hashed area at the top-left row of Figure 5. Then the WAVE device may switch to a SCH for a period called SCH Interval (SCHI) and is shown by the hashed area at the second row of Figure 5. Both CCHI and SCHI actual resource utilization are delayed after switching by a period called Guard Interval (GI) in order to accommodate for device differences. From Figure 5, it is clearly essential to minimize the GI by adopting accurate and efficient synchronization mechanism. As soon as a group of WAVE devices are synchronized, they alternate the utilization of the $\mathrm{CCH}$ and $\mathrm{SCH}$ as illustrated in Figure 5.

Among proposed synchronization mechanisms, two major approaches seem to gain popularity within the WAVE standards community. First approach allows WAVE devices to align their radio clock to the earliest clock signal the device receives. This distributed system has built-in robustness since roaming devices can adopt different clock reference as they move to newer communication zones. There are no concerns about nation-wide failure or fears of nation-wide attacks because any synchronization failure would be local to devices in a single communication zone. However, there is a little guarantee that WAVE devices cannot be led to follow invalid or malicious clock, which could be used to distribute obsolete security certificates. Furthermore the process of continuously drifting the clock results in lesser efficiency in terms of radio resource utilization.

The second approach assumes the availability of global clock signal with sufficient accuracy (like UTC). WAVE devices align their radio resources to a globally accurate clock every time period. A simple mechanism is employed to allow WAVE devices to align to the best available clock signal in the absence of a global signal like the situation when vehicles drive inside a tunnel for instance. This approach suffers from being too centralized. An attack or failure in the global clock source, while unlikely, leads to wide-spread irrecoverable failure of the DSRC networks. In addition, the impact of having clock signal that propagates through large geographical area at the start of each second remains unexplored. More research is needed to evaluate the impact of this clock signal on other forms of communications like satellite, cellular and other WiFi or WiMAX communications.

Current WAVE standards follow the global signal approach. However, it is likely that the WAVE adapts, eventually, a combination of the global signal and some other distributed approaches. In addition, it is important to decentralize the global clock reference and to initiate its signal differently based on geographic locations. Furthermore, preliminary results have shown that massive deployment of $5.9 \mathrm{GHz}$ can have some impact on other forms of radio communications. This issue, despite its vitality, remains largely unexplored and presents fertile research topics.

3.5. Reference Model. To clarify the fairly complex DSRC design we present a simplified reference model by dividing major WAVE architecture into blocks. From a layered perspective, WAVE devices employ almost common ISO/OSI stack as displayed in Figure 6. The figure illustrates a common communication stack and identifies the related standard for both data and management planes [15].

In Figure 6, we intended to avoid meandering details and conserve the core model ideas. A typical communication stack can be seen as two major planes, data plane and management plane. Data plane is focused on processing of 


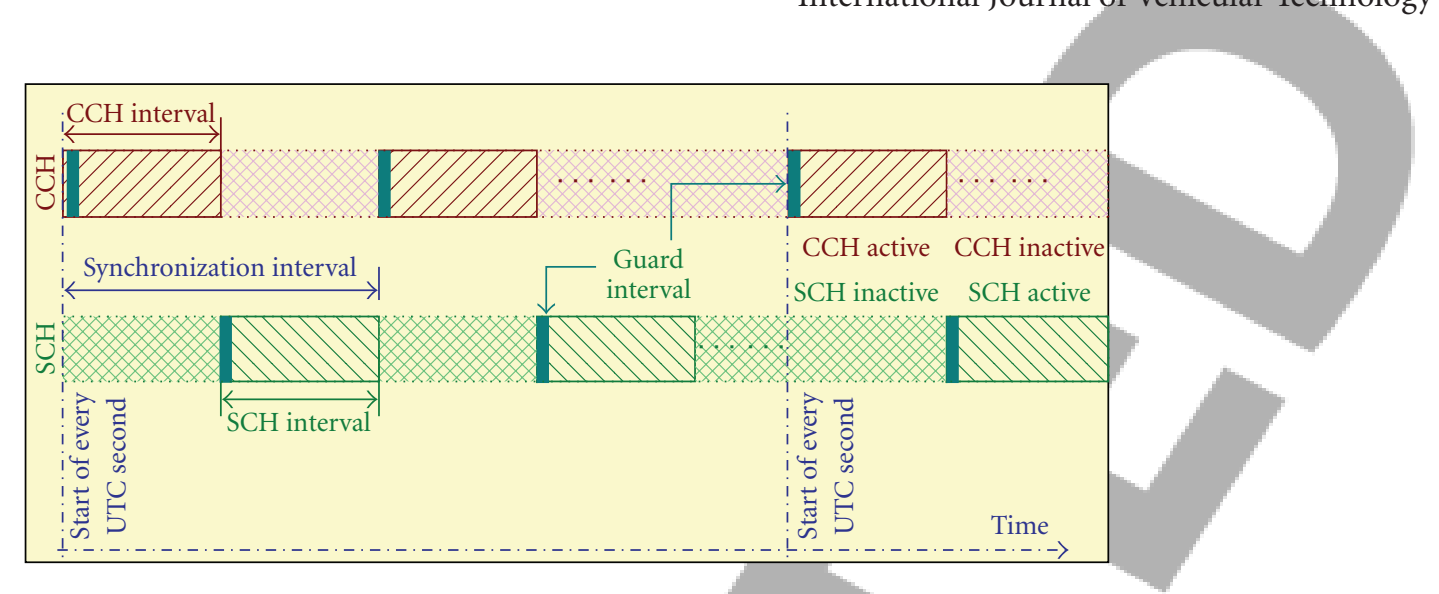

FIgure 5: WAVE Channel Synchronization.

data like adding or removing frame headers. Data Service Access Points (the green SAPs on the right in Figure 6) define formal interfaces between different data stacks. Management plane is focused on communication commands such as synchronization, channel switching. Sizable part of WAVE standards are centered on defining Management Service Access Point (the red SAPs on the left in Figure 6) for each entity. Standardizing SAPs allow plugging different entities. For instance, the SAP between WME and WSE as shown in Figure 6 is defined in both 1609.3 [8] and 1609.2 [11].

In Figure 6, blocks highlighted in light blue represent the focus of the WAVE standards. Blocks highlighted in dark blue represent WAVE amendments to other standards. The green blocks in the top right represent common WAVE interfaces with established standards outside the DSRC. For simplicity, WAVE applications are not presented in Figure 6. WAVE applications use common UDP/TCP interface stack.

It is important also to highlight the WAVE Short Message Protocol WSMP since it is unique to DSRC. WSMP packets may require special services like being transmitted using a particular power or data-rate. These unique requirements impose challenges to the WAVE-MAC layer and below. The MAC and PHY layers must test the contents of each packet to adjust radio power and data-rate before each packet transmission $[15,16]$.

The WAVE Management Entity (WME) represents another entity that is unique to WAVE standards and performs much of the operations unique to WAVE standards. For instance, when data frames are scheduled, the transmission channel must be defined along with QoS priorities. Those priorities must allow an emergency safety message to be transmitted at anytime with very limited latency. Management of frame queuing, priority channels and handling of safety messages are quite unique to WAVE standards. The WME handles those particular processing in coordination with other design entities.

The WAVE Security Entity (WSE) provides management of data encryption mechanisms and key management. The WSMP also takes part in enforcing security policies besides monitoring traffic patterns and responding to possible attacks [8].

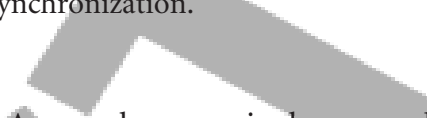

A sample scenario here would describe how different vendors may implement and build WAVE devices. For instance, one vendor might implement the blue boxes then use an of-the-shelf stacks as illustrated by the green boxes of Figure 6. The stack illustrated in Figure 6 also facilitates the building of devices that employ cellular and WAVE at the same time.

\section{WAVE MAC Services}

The following subsections illustrate the MAC layer services of the WAVE communications stack. The focus is on WAVE QoS and MAC queues operations, Channel Coordination between different WAVE devices, and describing the different Types of WAVE Communications. WAVE QoS and channel operations are fundamental to understanding and utilizing WAVE stack.

4.1. Quality of Service (QoS). This subsection is focused on WAVE QoS by introducing two main concepts, namely channel routing and channel selector. Then the section discusses the use of user priority to apply WAVE QoS. Finally, we discuss the main issues relevant to WAVE QoS.

WAVE QoS captures the fundamentals of 802.11e and extends it to cover two channel operations. As illustrated in Figure 3, the WAVE and $802.11 \mathrm{p}$ follow the 802.11e Enhanced Distributed Channel Access (EDCA) QoS paradigm. One of the major additions in WAVE networks is the transmission of WSMP packets. For each WSMP packet the WAVE standards dictate that it should be transmitted using:

(1) data-rate defined in-packet,

(2) channel number defined in-packet,

(3) transmission power defined in-packet.

The WAVE design extrapolates the 802.11e EDCA architecture as illustrated in Figure 7 to service both IPv6 and WSMP packets. For simplicity, Figure 7 avoids details of management and signaling traffic.

The reference architecture for WAVE MAC is distinguished from the 802.11e architecture by implementing Access Category queues on a per-channel basis and by the 


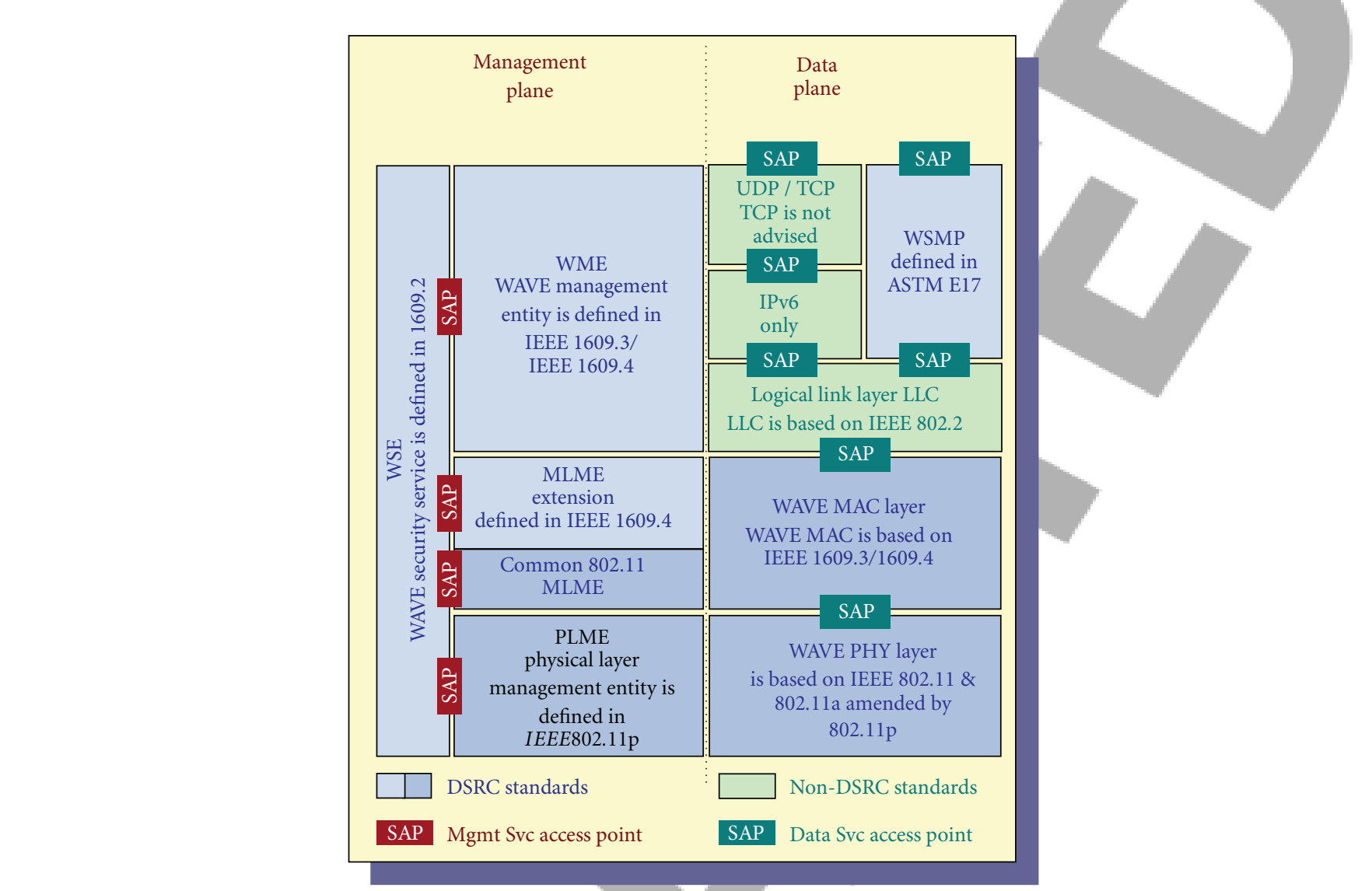

Figure 6: Simplified WAVE Communication Stack.

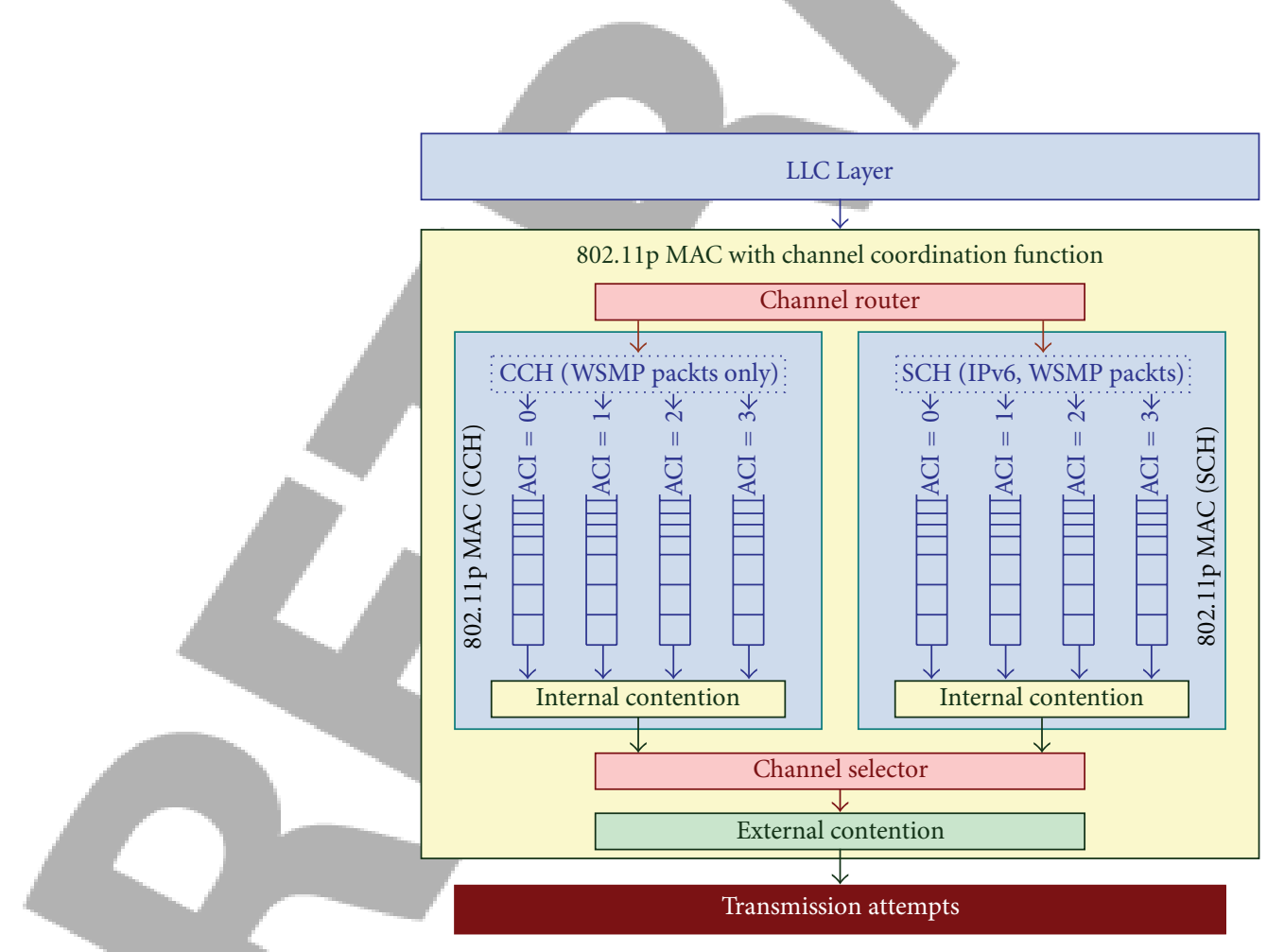

Figure 7: WAVE MAC-QoS Architecture. 
addition of Channel Coordination Function (CCF). The CCF implements a Channel Router and a Channel Selector as follows.

4.1.1. Channel Routing. The Channel Router detects the arrival of a WSMP datagram by checking the EtherType field of the 802.2 header. The Channel Router then forwards the WSMP datagram to the correct queue based on the channel identified in the WSMP header and based on packet priority. If the WSMP datagram is carrying an invalid channel number, the packet is discarded without issuing any error to the sending application.

The IP datagram transmission is slightly different. Before initializing IP data exchanges, the IP application registers the transmitter profile with the MLME (check Figure 6). The transmitter profile contains SCH number, power level, data rate and the adaptable status of power level and data rate. When an IPv6 datagram is passed from the Link Layer Control (LLC) to the Channel Router, the Channel Router routes the datagram to a data buffer that corresponds to the current $\mathrm{SCH}$. At any given time, there is only one active transmitter profile in a WAVE device. If the transmitter profile indicates specific SCH that is no longer valid, the IP packet is dropped and no error message is issued to originating application.

4.1.2. Channel Selector. Channel Selector carries out multiple decisions as to when to monitor a specific channel, what are the set of legal channels at a particular point in time and how long the WAVE device monitors and utilizes a specific channel. The Channel Selector also decides to drop data if it is supposed to be transmitted over an invalid channel like the case when a channel does not exist any longer. The set of policies enforced by Channel Selector can be fairly complex. Those policies are defined and communicated to the Channel Selector through the WME as indicated in Figure 7. The IEEE 1609.4 provides the detailed Management Information Base (MIB) identifying policy handlers.

4.1.3. User Priority. The concept of priority can be used in various ways. Applications have an application priority level, which is used by Networking Services to help decide which application gets preferred access to the communication services, that is, which application's WBSS to announce/join in case of a conflict. A different concept is the priority assigned to network data traffic. The lower layers use a separate MAC transmission priority to prioritize packets for transmission over the wireless medium. IP packets are assigned the MAC priority associated with the traffic class of the generating application. The MAC priority for WSM packets is assigned by the generating application on a packet by packet basis.

The general architecture of prioritized access for data transmission on one channel is shown in Figure 7. Upon the arrival of a datagram to the Channel Router, it forwards the datagram to the appropriate channel and data queue. The appropriate priority queue is selected by mapping the User Priority (UP as defined in transmitter profile) to an Access
Category Index (ACI). The Channel Selector schedules data for external contention by dequeuing priority queues based on their ACI. The Channel Selector also configures and confirms the media use of desired channel information.

4.1.4. Issues and Lessons Learned. It is imperative that endto-end QoS requires more than MAC QoS. MAC QoS mechanisms are focused on per-hop prioritization and cannot optimize the process of selecting data route to destination. To illustrate this point more, a comprehensive QoS solution would include a MAC layer QoS which operated on a per hop bases as illustrated here. Then the IP-QoS will define and monitor QoS through a particular path using common technologies like MPLS for instance. Then a third layer is needed on a per domain bases where QoS is defined between the domains' ingress and egress as in the DiffServ model. Finally another solution is required to monitor and manage end-to-end QoS. The reader is encouraged to augment his knowledge of the MAC QoS mechanism described here with intradomain mechanisms like in [17] in addition to cross-domain mechanisms like in [18] in order to shape a comprehensive QoS solution.

During the early design stages of the WAVE standards, we relied on 802.11 e and 802.11 hours without modifications. The resulting standards were convoluted and packed with inapplicable clauses. The DSRC workgroup decided to capture the essence of 802.11e QoS, 802.11 hours channel coordination yet maintained flexible design relevant to its operational environment. This approach resulted in the design of both channel router and channel selector. The algorithms that manages channel routing and channel selector remain hardly explored. Most of the research and publications on 802.11e QoS cannot be applied here without major redesign, mainly because optimum queuing on one channel is suboptimal when we change channels. Also the requirement of having predefined delivery time for all safety messages requires the design of an algorithm that guarantees delivery in deterministic, rather than statistic, manner. The 802.11p presents rich tools for researchers to optimize channel utilization by fine-tuning the policy parameters of the Channel Coordination MIB.

A sample scenario here could be a vehicle approaching an intersection with possibility of fatal collision. Concurrently, other vehicles are occupying the $\mathrm{SCH}$ and making a credit card transaction. The core objective of the presented QoS mechanism is to allow uncompromised and complete exchange of safety messages to prevent the fatality. Yet, the comprehensiveness, fidelity, and integrity of the credit card transaction should not be compromised.

4.2. Channel Coordination. This subsection introduces WAVE channel coordination and extends that to describe WAVE-MAC synchronization parameters and tolerance. In order to do that, we describe common time-based estimation. The subsection ends with a discussion of the main issues pertaining to channel coordination.

WAVE devices are expected to be implemented as either single-channel WAVE device which exchanges information 
and/or listens to only one radio channel at a time; or as multichannel WAVE device which exchanges information and/or listens to at least two channels at a time. In a WAVE environment, single-channel and multichannel WAVE devices may also decide to remain on the $\mathrm{CCH}$ ignoring the $\mathrm{SCH}$, but cannot ignore the $\mathrm{CCH}$. Thus, the WAVE environment identifies a time period called $\mathrm{CCH}$ Interval (CCHI) and all WAVE devices members of the same WBSS are scheduled to listen and utilize the $\mathrm{CCH}$ during the common (CCHI).

Figure 8 shows both the $\mathrm{CCHI}$ and $\mathrm{SCH}$ Interval (SCHI). WAVE devices may decide to ignore the $\mathrm{SCH}$ and remain on the $\mathrm{CCH}$ even during the SCHI. Therefore, it is imperative that WAVE devices within the same WBSS sustain accurate synchronization over time.

When a WAVE device joins a WBSS, it listens to the $\mathrm{CCH}$ until it receives the service announcements which contain both CCHI and SCHI. The values of CCHI and SCHI can be formed as follows:

$$
\text { Sync. Interval = CCHI }+ \text { SCHI. }
$$

At the start of each scheduled channel interval, a Guard Interval (GI), shown in Figure 8, is used to account for variations in timing accuracies among different devices. WAVE devices cannot transmit during the GI. To prevent devices from attempting to transmit simultaneously at the end of a GI, a medium busy is declared during the GI so that all transmission attempts are subject to a random back-off at the start of each channel interval.

At the scheduled channel interval, all prioritized MAC activities on the previous channel are suspended and the prioritized access activity on the currently scheduled channel starts or resumes if they were suspended. Meanwhile, the Channel Coordination Function prevents packets from being transmitted on the incorrect channel.

A good implementation would consider avoiding transmission at scheduled GI. For instance, if the expected MSDU (a single MAC Service Data Unit) transmission time is more than the time remaining before the next GI, then the transmission can be avoided and the MSDU can be buffered for next channel interval. Optimizing Channel Coordination Function (CCF) is a complex discretion. For instance, [19] proposed the use of cross-correlation technique to detect the starting point of a short training symbol. Guard Interval's starting position of long training symbol is then found by reapplying the same technique. Such a technique improves robustness in high-mobility low signal-to-noise ratio conditions.

Finally, we must indicate that the time synchronization is a critical issue in the WAVE channel coordination. The impact of GI on the efficiency and throughput is not completely investigates. A numerical study could elaborate on how errors in synchronization affect performance and throughput. These types of studies are much needed to evaluate potential delays in crash situations. Another area for empirical studies is evaluating the time required to reset channel parameters, such as power-data-rate and channel, to deliver safety messages. The suggested research areas here represent topics we plan to investigate in the short term.

4.2.1. Channel Coordination Function. A WAVE device can only be a member of one WBSS at a time. The IEEE 802.11 active scanning is prohibited in $802.11 \mathrm{p}$. Multichannel WAVE devices monitor $\mathrm{CCH}$ at all times, but single-channel WAVE devices must implement Channel Coordination Function in order to monitor $\mathrm{CCH}$ at all common CCHI.

The WAVE standards assume the availability of an external accurate common time reference. WAVE devices utilize the IEEE 802.11 Timestamp Field (TSF) along with a WAVE specific Timing Information Field as inputs in estimating UTC time. The value of the IEEE 802.11 TSF is an integer modulus $2^{64}$, incremented in units of microseconds [9].

4.2.2. Synchronization Parameters. The Coordinated Universal Time (UTC) is a relative time measure compared to instant 2004-01-01T00:00:00.000000 as in [9]. UTC is available in most GPS devices. A typical GPS-UTC precision, at the time of this paper, is at least 1 PPS (pulse per second) UTC signal (with error $<100 \pm$ nanoseconds) [16]. Experimental trials confirmed that 1 PPS signals are sufficient to synchronize multiple WAVE device timing.

WAVE devices use the UTC time to form TSF. The TSF timer plus any offsets estimated, which resolves to an integer number, is incremented in units of microseconds, and is used as the WAVE device's internal estimate of UTC time. Singlechannel WAVE devices use this UTC estimate to determine when the device exchanges data on SCH. Single-channel WAVE devices continuously monitor the $\mathrm{CCH}$ when not synchronized to UTC and when joining a new WBSS. It is important to highlight here that the use of UTC is entirely optional. Other sources of universal time reference can be 


\begin{tabular}{|l|l|l|}
\hline $\begin{array}{c}\text { biming } \\
\text { capabilities }\end{array}$ & $\begin{array}{c}\text { TSF timer } \\
\text { offset }\end{array}$ & $\begin{array}{c}\text { TSF timer } \\
\text { standard deviation }\end{array}$ \\
\hline & & b octets $\longrightarrow$ 3 $\longrightarrow$ 3 \\
\hline
\end{tabular}

FIGURE 9: Timing Information Field.

used as long as the required accuracy is maintained [9]. Some of the critical research issues related to synchronization can be found in [19]. The end of this subsection incorporates further discussion of WAVE timing accuracy in terms of relevance to vehicular applications [16].

4.2.3. Common Time Base Estimation. Single-channel WAVE devices can synchronize to UTC time by implementing an estimator of UTC time as described before and by implementing an estimator of the standard deviation of the error in the UTC time estimate. Standard deviation is defined as the square-root of the second moment of the probability density function of the estimation error. Note that this includes the effect of any biases in the estimate of UTC time. For example, GPS can be used as an input to a simple estimator that changes the GPS time reference to UTC 2004-01-01T00:00.000000, calculates the necessary TSF Timer Offset value, and sets the standard deviation to that of the standard deviation of the time output of the GPS device under the given operating conditions [16]. A slightly more complex implementation could use information from the Timing Information Field in received WAVE service announcement to update an internal estimator of UTC time along with an estimation error variance (standard deviation) as outlined hereafter.

The Timing Information Field is detailed in Figure 9. This field holds information that can be used by recipients to estimate UTC. The Timing Information Field includes the Timing Capabilities, a TSF Timer Offset, and TSF Timer Standard Deviation subfields.

The Timing Capabilities subfield identifies the timing capabilities of the initiator WAVE device in terms of being single/multichannel, having generated UTC through a GPS, and having continuous time source availability.

The TSF Timer Offset subfield contains the 2's complement integer of the TSF timer offset in microseconds, which when added to the WAVE device's TSF timer value generates the WAVE device best estimate of UTC time at the instant when the first bit of the WAVE service announcement is transmitted from the WAVE device's antenna connector. The TSF Timer Standard Deviation subfield is an unsigned integer of number of microseconds. This number represents the estimate of the standard deviation of the UTC error in the WAVE device's estimate of UTC time. When the TSF Timer Standard Deviation subfield is set to its maximum value (224), it indicates that the value of the TSF Timer Offset subfield is meaningless.

The value of the TSF Timer Standard Deviation and the TSF Timer Offset are function of various environmental and implementation factors; and may vary with time. At

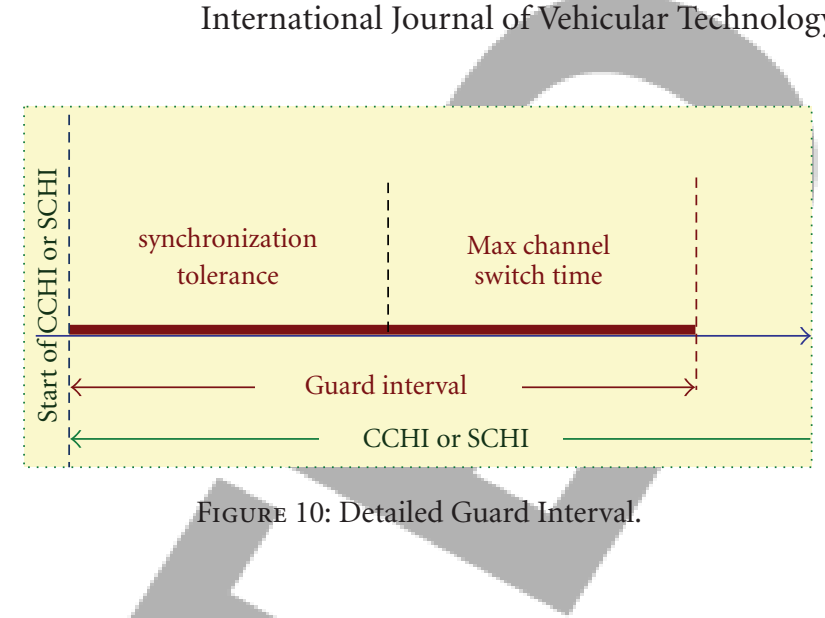

startup, the TSF Timer Offset is set to zero and the TSF Timer Standard Deviation is set to its maximum value to indicate that the TSF Timer Offset is currently invalid. Once a lower variance source of UTC time becomes available, the new source and its error variance are adopted as initial conditions for the UTC estimator.

4.2.4. Synchronization Tolerance. Figure 8 illustrates the synchronization timeline in WAVE environment. Each CCHI and SCHI starts by a GI (Guard Interval). Figure 10 details the GI to be the sum of Synchronization Tolerance and the Max Channel Switch Time. It is important to indicate that the figure is not to scale. The GI is typically a small fraction of the CCHI or SCHI. The Synchronization Tolerance is defined to be double the $95 \%$ probability threshold value that determines whether a WAVE device is synchronized to UTC. The Max Channel Switch Time is the maximum time the WAVE device takes to switch channels.

Since local device clocks at two different devices may drift in opposite directions. WAVE devices are defined to be synchronized to UTC if it complies with the following condition:

$$
3 \times \text { TSF Timer Std Dev }<\frac{\text { Sync.Tol. }}{2} .
$$

Single-channel WAVE devices that are not synchronized to UTC monitor the CCH continuously, do not offer services on SCH, and do not act as a Service Provider. If a WAVE device is a member of a WBSS and is processing a transaction on SCH at the time when synchronization is lost, the device simply discontinues the ongoing transaction and reverts to monitoring the $\mathrm{CCH}$.

4.2.5. Issues and Lessons Learned. Timing accuracy presents a cornerstone in the development of the WAVE standards. Roaming users are authenticated by presenting a security certificate that has a limited time span. A simple attack may copy a certificate, alter it, and reuse towards malicious objective. The limited time span of a certificate is essential to secured communication. The multiples of microsecond accuracy may be acceptable for current computing devices but it is inevitable that higher accuracy will be needed in the future.

Time synchronization is critical to WAVE channel coordination. Evidently, this section included sufficient details 
on the fairly complex WAVE synchronization mechanisms. The complexity, though, emanates from the initial decision to interoperate both single and multiradios. This decision is based on the current availability and cost of short-range radios. In a forward look to the future version of the WAVE standards beyond the "trial use", one may assume the use of multiradio that can listen to $\mathrm{CCH}$ while communicating on one or more $\mathrm{SCH}$. This approach not only simplifies the design, it improves channel utilization by the following percentage:

$$
\text { Enhancement } \%=\frac{(\mathrm{CCHI}+2 \mathrm{GI})}{\text { Sync. Interval }} .
$$

Another issue in the current design is the fact that it utilizes limited band of the assigned spectrum at any point in time/location while leaving the majority of the assigned band unutilized. In an ideal design, a roaming multiradio $\mathrm{OBU}$ may listen to $\mathrm{CCH}$; communicate with an RSU on one $\mathrm{SCH}$, while communicating with other OBUs on many different SCHs. It is especially intriguing to think of an adaptive algorithm that allow OBUs to dynamically and autonomously locate and utilize different $\mathrm{SCH}$ based on sensing resource availability. We believe that developing such an algorithm facilitates the development of much alluring applications, especially V2V applications [20].

Then, another challenge may seem a little peculiar at first. A short-range wireless technology like WAVE may need to be carried over long-range communications like cellular. Certainly, the basic concept of DSRC is to provide high bandwidth communication configured for localized services and traffic safety use. The concept of short-range communications supports the public safety applications perfectly fits most of the situations but not quite for all road situations.

Long highway stretches that span multiple miles with little difference in traffic information and limited traffic volume present a good example for situations when DSRC is uneconomical. In those situations, using WAVE devices leads to the unnecessary deployment of massive infrastructure. Instead, lesser number of cellular services can be deployed to carry the DSRC communications using WAVE standards over a cellular media for example. In other words, the implementing WAVE over different physical layer, but allow OBUs to pick up the signal of the other layer (like cellular) and then process it like MAC layer processing of any other WAVE device.

In order for this proposal to work, OBUs must be equipped with multichannel that utilizes cellular signals. A better approach is to abstract the DSRC WAVE layer using SDR like the one proposed in $[21,22]$. This approach, while complex, realizes the inevitable use of SDR to provide support for applications running over multiple vertical radio layers.

A sample scenario of channel coordination is a situation when low-end devices that are unable to switch channels communicate with high-end devices that might utilize one or more service channels communicate. In that scenario, the low-end device might stay on $\mathrm{CCH}$, while the high-end device must leave $\mathrm{SCH}$ and visit $\mathrm{CCH}$ at the right time to receive safety messages at the time when it is being broadcast.

4.3. Communication Types. This subsection introduces WBSS versus non-WBSS operations which can be mapped to vehicle-to-roadside versus vehicle-to-vehicle communications. In this subsection we also define WAVE communication service types and WBSS initiation, operation and termination. Finally, we discuss the management of changes to WBSS operations. The subsection ends with discussion of the main issues pertaining to WBSS and communication types. This subsection is also a necessary read before discussing WAVE service management.

WAVE communication services provide data communications over two protocol stacks, namely; IPv6 and WAVE Short Message Protocol (WSMP). WSMP is unique to the WAVE standards and is designed for use by specialized applications like safety applications. Applications using WSMP may initiate a WBSS to configure the SCH for their use. But availability of $\mathrm{SCH}$ is optional since WSMP can be exchanged on the $\mathrm{CCH}$ even in the absence of WBSS (i.e., in a V2V scenario).

4.3.1. WBSS versus Non-WBSS Operations. While the use of WBSS is expected to be dominant in DSRC networks, it is not necessary the case. WAVE devices can communicate WSMP messages over WAVE networks without WBSS. A scenario involving WSMP use without WBSS goes like this.

(i) A source WSMP application registers with the WME, then, composes WSMP data for transmission, and addresses WSMP data to a broadcast MAC address. The MAC reads the required channel information (power level, data rate) from the packet header and set the $\mathrm{CCH}$ for transmission using the channel parameters as requested (power level, data rate). Transmission takes place based on both internal contention and media contention.

(ii) A receiving device accepts the packet and passes it up the communication stack. The WSMP stack delivers data to the locally registered application, based on the Provider Service Identifier (PSID) and on the Provider Service Context (PSC). At this point, the receiving application knows the availability and address of the transmitting application, and can continue the exchange on the $\mathrm{CCH}$ if desired, using either unicast or broadcast MAC addresses, as appropriate.

When operating with a WBSS, a WAVE device initiates the WBSS at the request of any application running on (or through) the same device. The WAVE device initiates the WBSS on the CCH of the provider [8]. Details on the use of PSID and PSC are described in Section 5 as part of the WAVE service management.

4.3.2. WAVE Communication Service Types. WAVE supports two types of communication services; namely persistent and nonpersistent services. The distinction being that a persistent 


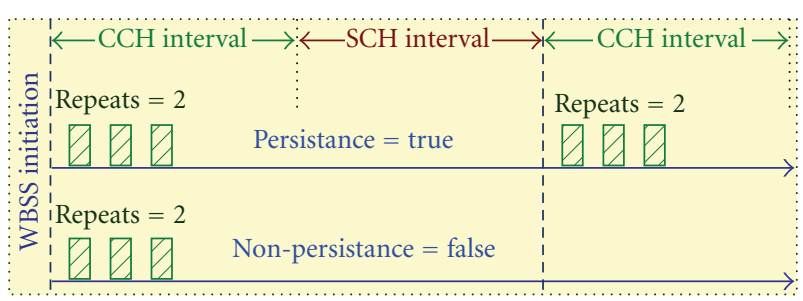

Figure 11: WAVE Persistent Communication Service.

WBSS is announced each CCH interval, and a nonpersistent WBSS is announced only on initiation. A usage of the persistent WBSS would be to offer an ongoing service to any devices that arrive into range like the case of roaming OBU. A usage of the nonpersistent WBSS would, typically, be to support an on demand service like the case of downloading files while waiting in a parking lot.

As illustrated in Figure 11, a persistent WBSS is announced periodically, and could be used to support an ongoing service for indefinite duration, such as general Internet access. Persistent WBSS communication service resembles the normal vehicle operations on the road. A nonpersistent WBSS is announced only on WBSS initiation, and might be used to support a WBSS with limited duration. Both persistent and nonpersistent operations use repeat announcements, but only Persistent WBSS rebroadcast the repeats each $\mathrm{CCH}$ interval. A practical example of nonpersistent service is a garage service where a consumer vehicle can join a private WBSS to download files or upgrade software. Typical examples show stationary units forming a WBSS for a long term relation [8].

In WAVE communication services, WAVE devices may take the role of either provider or user on a given WBSS. This is determined by the role chosen by the application operating on the device. The provider device generates announcements to inform other devices of the existence of the WBSS, and the presence of the associated application service(s).

The user role is assumed by devices that join the WBSS based on receipt of the announcement. A device may change its role when it participates on a different WBSS. The terms provider and user do not imply any particular behavior of the applications once the WBSS is initiated or joined. A device can be a provider for one service and a user of another.

4.3.3. WBSS Initiation and Operations. A WBSS is initiated by the WAVE Management Entity (WME Figure 6) when a provider application redefines some of the WBSS parameters like the persistence status. The announcing WAVE device forms the Provider Service Table PST. As soon as a user application starts on a WAVE device, it must register all services it might need with the local WME. Upon the receipt of a WAVE service announcement, the receiving WME checks whether the provider application, defined by the Provider Service Identifier (PSID) in the announcement, is of interest to any locally registered user applications. User applications have the option to be informed of announcement containing both
PSID match. When a match is found, the WME takes one of two actions, depending on the user application registration parameter. In the simple case, the WME generates the necessary MAC primitives to cause the local device to join the announced WBSS, by tuning device to the correct $\mathrm{SCH}$ at the correct time, and by setting any other lower layer configuration parameters appropriately to support the communications. Alternately, the user application may choose to reconfirm before joining of the announced WBSS. This gives the user application an additional control level allowing any application to decline participation in specific service if it has recently accomplished some milestone objectives.

Upon decision to join the announcing WBSS and as soon as the communication parameters are set successfully, the WME sends a notification to the local user application. Subsequently, the user application is free to generate WSMP or IPv6 data packets for transmission on the SCH. Received packets are delivered up the WSMP or IPv6 stack. The WBSS stays in place at the local device until it is terminated [8].

4.3.4. WBSS Termination. Once initiated, a WBSS stays in place at each participating device until locally terminated. WAVE devices may independently decide to leave a WBSS. There is no protocol exchange over the air interface to confirm the end of a WBSS. The WAVE device WME may decide to issue a request to its MAC layer to leave a WBSS and to inform all the affected applications through a notification (i.e., process WBSS termination) in any of the following situations.

(i) All applications indicate the completion of their activities, via a request changing their status.

(ii) Participation on a conflicting WBSS (e.g., on another channel) is required to support a higher priority application.

(iii) The lower layer indicates the $\mathrm{SCH}$ has been idle for a predefined amount of time, implying an irrecoverable loss of the WBSS.

(iv) The security credentials associated with the WBSS announcement expire at the user or are determined to be invalid when checked.

4.3.5. Changing WBSS Services. In a nonpersistent WBSS, a provider application that is registered after issuing the initial WBSS cannot join the ongoing WBSS services.

On the other hand, persistent WBSS offers different sets of applications over time by altering the announced Provider Service Table (PST) during different $\mathrm{CCH}$ interval. To support the dynamic PST feature, the announcement's destination MAC address is constrained to be the broadcast address. Applications come and go from the provider's WBSS as triggered by the application request primitive. The provider WME may also end a persistent WBSS as described in the previous subsection. 
User applications start and stop participation on a persistent WBSS during its existence. The WME maintains a table of active/inactive status of each participating application. The WME of the user WAVE device joins and ends local participation on the WBSS as required based on the user applications' status [8].

4.3.6. Issues and Lessons Learned. WAVE standards workgroup have decided to adopt the use of IPv6 as a network layer protocol. The decision is fueled by the massive demands the DSRC environment imposes on the WAVE standards design. The number of roaming vehicles demanding IP addresses is simply beyond the IPv4 capabilities. Further, IPv6 approach to managing mobility is much favorable compared to IPv4.

The WAVE standards also uses both WBSS and nonWBSS operations. Non-WBSS operations should not be confused with the Independent Basic Service Set (IWBSS) which supports decentralized wireless ad hoc networks. It is widely believed that a new WAVE-IWBSS operational mode is required to facilitate fast, adaptive, self-configuring V2V communications. Regrettably, current WAVE standards do not provide any design mechanisms in response to such aspiration. That ambitious is shared with other stakeholders and we believe WAVE-IWBSS mode will be part of next WAVE efforts. The IETF attempted to synchronize the development of both NEMO and MEXT standards with WAVE standards. NEMO (Network Mobility) and MEXT (MAC Layer Management Entity) projects were established to resolve issues pertaining to mobility. Researchers must pay attention to the current limitation and propose competitive solutions. One common disclaimer remains important since the WAVE standards do not preclude the use of point-topoint mechanisms towards V2V communications [20].

WAVE service types have been developed with IntelliDrive architecture and applications in mind. The ideas of persistence and termination of WBSS are highly relevant to the vehicular environment. Both mechanisms show how stringent environments impose specific design constructs.

Another issue of major concern here is scalability. The expected size of DSRC network is high enough to raise scalability concerns. The, interference with non-DSRC devices magnifies scalability concerns even further. Up to this date, there have been almost no publications on the issue of DSRC scalability and load balancing. The reader is encouraged to look at [23] and the scalability research on ad hoc networks as it has closer resemblance to vehicular networks.

Final comment here is relevant to IP address assignment and autoconfiguration. While IP addressing is relevant to the general WAVE architecture, it is important to realize that both WAVE and $802.11 \mathrm{p}$ are Link Layer Control standards. Mechanism described in WAVE 1609.3 should be treated as mere recommendations, and users may or may not follow it without violating the standards. We refer the reader to $[24,25]$ for more mechanisms designed for the WAVE environment.

\section{WAVE Services Management}

This subsection described WAVE service management which elaborates WAVE approach to managing services and providing applications with information on available services, in addition to enforcing policies. The following subsections describe the process for application registration, WBSS management, and join policies. Then describe the WBSS status transition and maintenance. We also describe how channel activities are being monitored. Finally, we discuss the main issues pertaining to WAVE service management.

As a general rule, the WAVE standards prohibit unregistered applications from gaining access to WAVE services. This rule improves WAVE security level compared to common WiFi standards. The few exceptions to this rule are explained hereafter.

Let's define a provider device as a WAVE device that has legitimate applications registered to offer WAVE services and is also the initiator of a WBSS. A provider WAVE device is a sender of WSM. Then, define a user device as a WAVE device that has legitimate applications registered to use WAVE services and is also the joiner of a WBSS. User WAVE device is a receiver of WSM.

A Provider-Service-Table (PST) is a collection of data describing all applications registered with a WAVE device to, legitimately, offer services. Provider applications must register with the WAVE device using its Provider-Service-ID (PSID). Each provider WAVE device builds its own PST and maintains it over time.

Similarly, a User-Service-Table (UST) is a collection of data describing all applications registered with a WAVE device to, legitimately, use services. User applications must register with the WAVE device using its User-Service-ID (USID). Each user WAVE device builds its own UST and maintains it over time.

A provider WAVE device announces its PST. When a user WAVE device locates the provider's WBSS it extracts the provider PST; and then, the user WAVE device compares the set of PSID available in the provider PST with its own UST that is available through its WME and contains local USID of applications demanding service. The user WME generates a new table of applications with matching interests. This new table represents the set of applications that are allowed to have WAVE services.

Before we go any further, three notes are important here. First, the WAVE standards use the term PST to describe both PST and UST. Second, the WAVE standards use the term PSID when referring to both PSID and USID. We thought that distinguishing the terms into PST, PSID, UST, and USID alleviates confusion. Finally, the use of the term "legitimate" in this section indicates an exchange of signed security certificate. Section 5.4 illustrates the use of time-out mechanism to issue and revoke those certificates. To simplify the reading of this section, we use the term "legitimate" to show that some sort of authorization and authentication takes place. Similarly, some of the terms described hereafter are introduced to simplify reading. Our experience has shown that reading through the standards drafts and RFCs with cluttered cross references and legally driven clauses can 
be quite confusing. The approach we followed here simplifies reading, yet adheres wherever possible to the formal terms used by the WAVE standards.

The mechanism described hereafter illustrates how WAVE devices grant application access to WAVE services. The following subsections detail the WAVE service management in precise terms.

5.1. Application Registration and Removal. All applications must register, as a provider or user, with its WAVE device WME before gaining services. Applications registered on the same WAVE device must have a unique PSID. This rule is not limited to WAVE-applications; it applies even to typical IP-based data access. The only exception is that unregistered WSMP provider application may send WSMP packets. WSMP user application must be registered to receive any WSMP.

The WME maintains a record of all registered applications in an Application Registration Table (ART). In other words, the ART is merely the union of the local PST and the local UST

$$
\mathrm{ART}=(\text { local PST } \cup \text { local UST }) .
$$

Before accepting new application registration the WME confirms that the new application is unique and has the necessary security credentials to gain the required access to its WAVE media. Applications failing to satisfy the two conditions are denied registration and receive registration denial message. Otherwise, applications are registered and receive a confirmations message.

The ART table is maintained by the WME which monitors an application status field. The WME uses the application status field to monitor the active/inactive status. Other fields maintain operational information like the IPv6 notification address, port and priority. Any Application can remove its registration by sending a message to the WME. In such a case, the WME removes the application registration entry from the ART. If the ART contains no active applications the WME ends the current WBSS [8].

5.2. WBSS Management. Generally, all WBSS are established on the $\mathrm{CCH}$. But based on an application request, a WAVE device may establish the WBSS on a SCH, and announce its presence on the $\mathrm{CCH}$ in order for other devices to join that WBSS. All WBSS are initiated based on service priority and the availability of radio resources. A WBSS can be newly established or it can be a modification to existing persistent WBSS.

Another important table is the Application of Interest Table (AOIT). As soon as a user device discovers a new WBSS, it uses the remote PST it receives via WBSS and its local UST to generate the AOIT as follows:

$$
\text { AOIT }=(\text { remote PST } \cap \text { local UST }) .
$$

Since a WAVE device can be a member of only one WBSS at a time; a user device that is not a member of any WBSS joins a new WBSS if

$$
\text { AOIT }=(\text { remote } \mathrm{PST} \cap \text { local UST })>\text { Null. }
$$

The WME takes the decision to join a specific WBSS following the application status transition diagram illustrated in Figure 12. However, if the user device is currently a member of a specific WBSS it joins a new WBSS only if the new AOIT provides services with higher priority compared to old AOIT. A user device may decide to leave a WBSS if the currently active applications conclude, timeout, or lose security credentials.

The provider device transition to a new WBSS and the termination of old WBSS are illustrated in Figure 12. The WAVE applications may have the status of "Active" during the application communication session, "Inactive" while waiting for a communication session, and "Unavailable" when the application is temporarily unavailable, for instance, waiting for other application to generate an event. It is important here to highlight that the WME checks the availability of the requested radio resources, inspects the new WBSS security credentials, and validates other MAC Layer Management Entity (MLME Figure 6) requests before disrupting the current WBSS services and before seeking transition to newer WBSS. If the WME detects a failure in the transition to the new WBSS, it stays on the old WBSS and sends an error message to the relevant application indicating the reason code [8].

5.3. WBSS Join Policies. A WAVE device joins a new WBSS based on a set of policies that are stored and executed by its WME. Those policies are invoked after the WME compares the set of services received from a remote device through the new WBSS with its own set of desires services.

The logical flow diagram and policies of WBSS are simplified in Figure 13. The policies utilize Active Application Table (AAT) which is the subset of Application Registration Table (ART) that contains only applications conducting active communication session at any point of time. Applications remaining in active beyond certain timeout threshold are moved out of the AAT, following specific message exchange, but remain in the ART.

As soon as the device joins the new WBSS, the WME changes the status of relevant applications to "active." Multiple applications match with the same Channel Number share the same WBSS.

The basic algorithm of Figure 13 can be described as follows. When a WAVE device discovers a new WBSS it downloads the new AOIT and compares it with its own AAT. If there are applications in AOIT with higher priorities, the WAVE device will certainly be interested in sending a join_conf message to relevant applications, but before that, the WAVE device must terminate running applications with lower priority if those applications require a conf_before_join message. Finally, if newer applications accept and confirm interest in the new WBSS, the WAVE device stops the old WBSS, notifies all applications in the old AAT, and joins the new WBSS.

5.4. WBSS Status Transition and Maintenance. The WME uses the application status field of the ART to maintain the status of each registered application based on supporting 

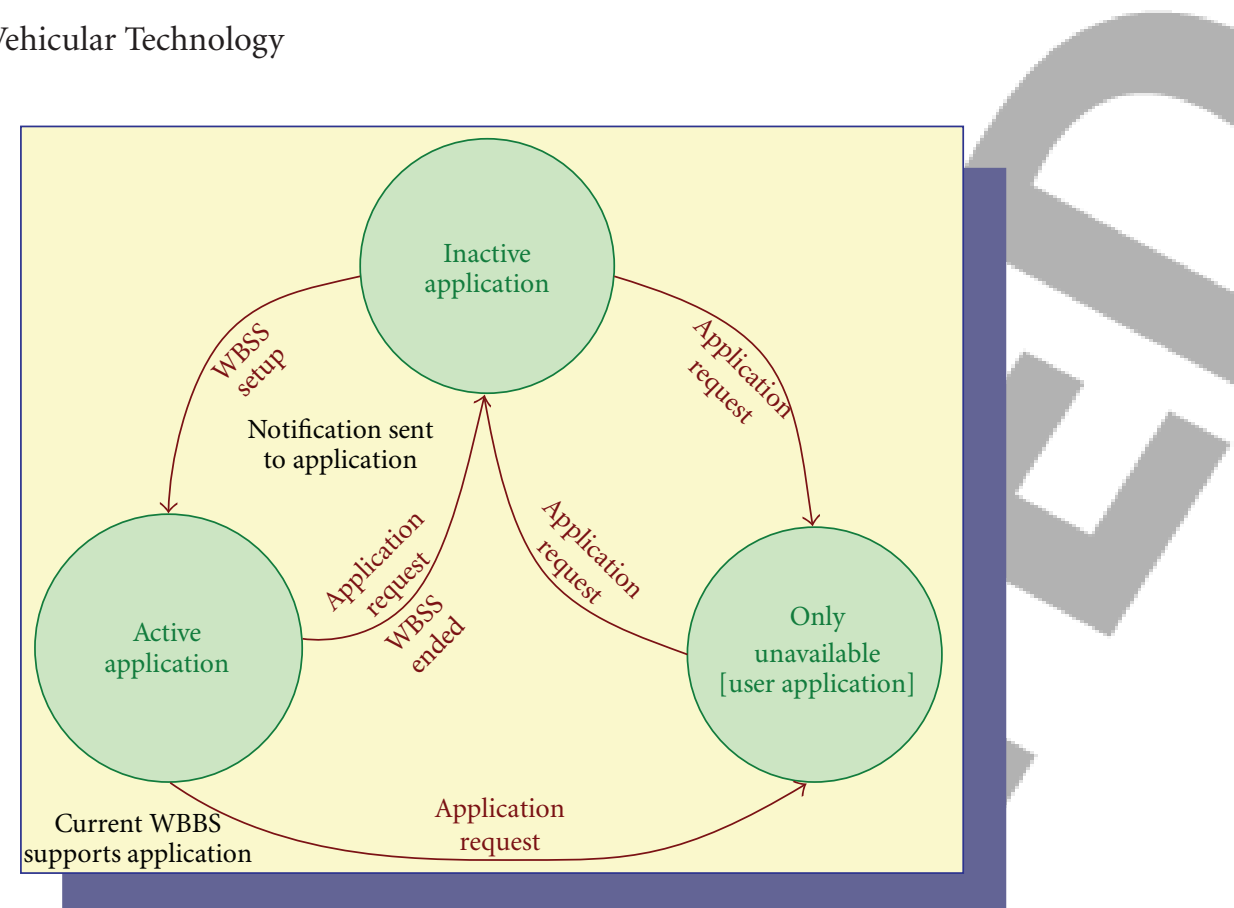

FIGURE 12: Application status transition diagram.

WBSS status and application availability. The default status of all applications is "Inactive". The WME attempts to match the remote PST with its local UST, and when a match is found, the WME may initiate a join WBSS as specified in Section 5.3 earlier. While operating on a WBSS, active applications may generate traffic for transmission on $\mathrm{SCH}$. The state diagram showing the WBSS status transition is illustrated in Figure 14.

A user application may choose to become unavailable instead of removing its registration. Unavailable status indicates a user application that is dormant for some time. One use of the unavailable status is the case when an application waits for an event to take place on different application before it is retriggered. The WME never joins a new WBSS, nor maintains a current WBSS on behalf of unavailable applications. Simply the WME treats unavailable applications like nonregistered applications.

5.5. Channel Activity and Usage Monitor. The MAC Layer Management Entity (MLME Figure 6) monitors channel inactivity and triggers internal indicator to the WME when the active $\mathrm{SCH}$ becomes idle longer than a prespecified time period. The WME assumes that a user device should cease operation on the current WBSS and proceed with WBSS termination.

The MLME also keeps track of all SCH in use by other devices within a listening range. Consequently, the WME can choose to operate on a SCH that is likely to have the least congestion whenever the WME is required to initiate communication on arbitrary SCH. The WME keeps track of the most recent time at which a WSA was received on each SCH (from the set 174, 176, 180, and 182). When the application requests the best available channel, the WME uses the $\mathrm{SCH}$ with the oldest received WSA [8].
5.6. Issues and Lessons Learned. The WAVE service management is, arguably, the most controversial part of the WAVE standards. Evidently, the idea of using provider service ID (PSID) as a unique service identifier has been used before with little success in Bluetooth 802.15 [26] and Bluetooth 2.0/2.1 [27]. WAVE standards define PSID to be a field of $2^{31}$ possible flat numbering services in order to accommodate future unprojected services. Nevertheless, services other than those described in the WAVE specifications cannot be supported. An example of that is a service that is generated in ad hoc manner due to immediate need.

Some issues here are of major concern. First is once a new service is invented, who would define the services and decide it doesn't conflict (in part or in full) with other services. Second, what would be the mechanism to distinguish valid services from bogus ones? Third, is how we can clean up services that no longer exist? The list of unresolved questions keeps rising.

The use of flat numerical codes to refer to services administrated by central authority leads to inefficient service discovery in networks with a large number of services. A simpler mechanism may use hierarchical service definition to improve service location by adopting a tree-like hierarchy and by narrowing services into groups of relevant family of services.

Hierarchical service approach facilitates the use of localized services where one type of service is available in certain municipality or local jurisdiction and absent everywhere else. Imagine a type of on-street gaming where players drive around trying to locate a treasure. Some city like Vegas may be interested in utilizing the WAVE $\mathrm{SCH}$ to provide players with clues. Other cities may strictly prohibit all gaming services. We believe that hierarchical services [28] fit naturally the dynamics of the DSRC environment and need to be accommodated. 


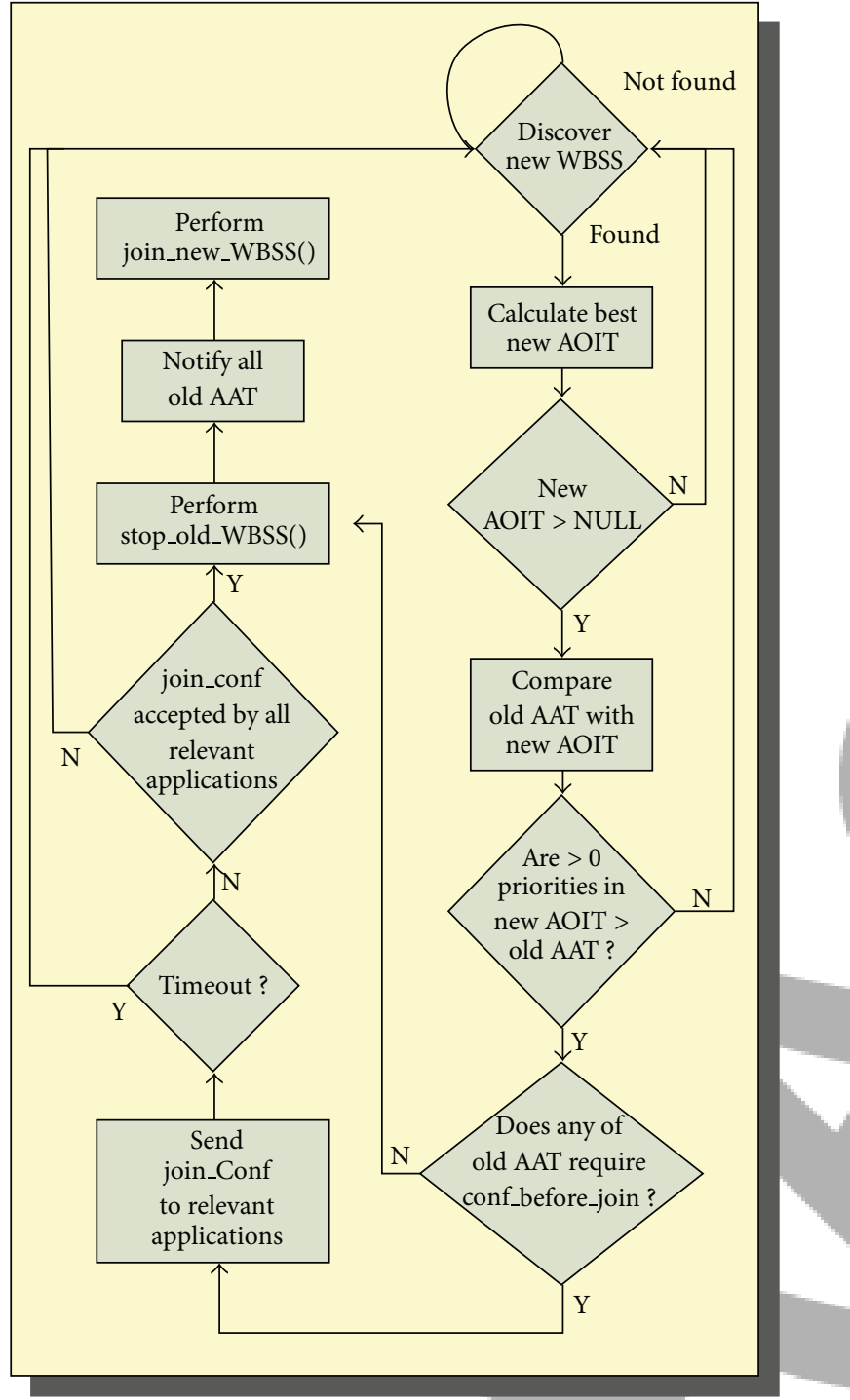

FIgURE 13: WBSS join policies and logic.

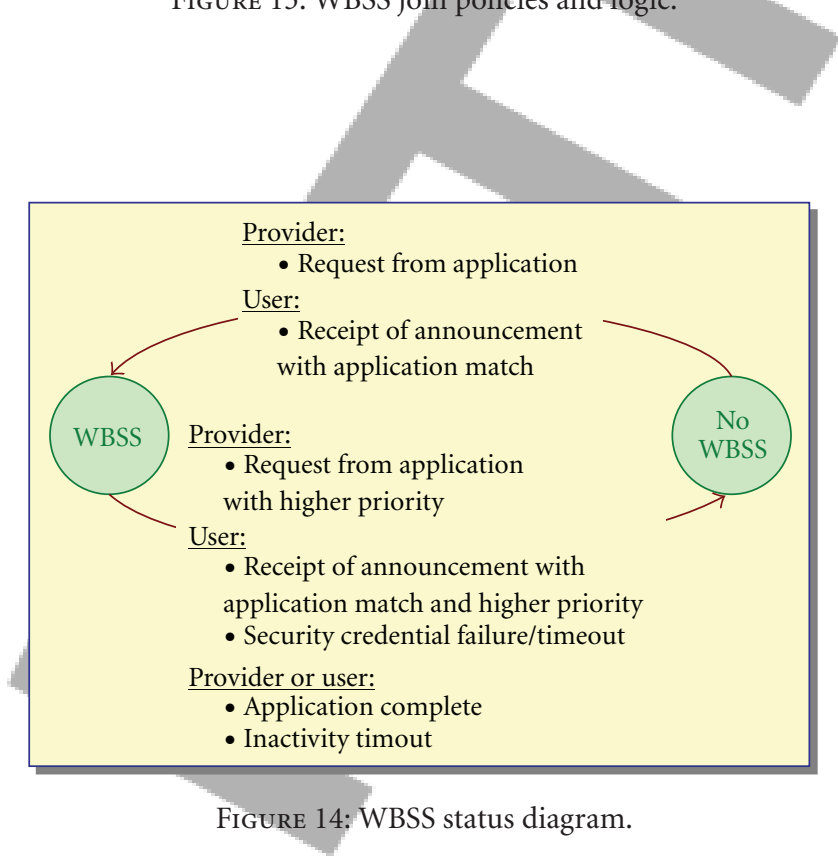

A simple sample scenario here could be a WAVE device that is running an application which demands particular services like toll-road payment. Once the vehicle arrives within the vicinity of a WAVE device that provide the required service, the toll application is informed and the transaction is carried out. A complex sample scenario would be an application that demands map information and credit card payment services. The reason is that one service may be available in particular vicinity, but not the other one.

\section{Conclusion and Future Directions}

This paper covers the current status of the WAVE standards. Most of the WAVE standard suite has been released for "trial use" and the rest is expected to be released by mid 2010 . The current IEEE 1609.x has been charted for "trial use" and the involvement of research community is highly desired for future DSRC development. Currently, much of the research on DSRC is spent on performance evaluation like in $[29,30]$.

This paper focuses on the fundamental mechanisms used in WAVE standards in response to the vehicular communication environments as defined in both DSRC and IntelliDrive towards, primarily, enhancing road safety. We extended the discussion in areas relevant to MAC services, QoS and service management. We described the use of channel coordination, WAVE communication types and WBSS policies in plain language. We guided the reader into specific subjects that require further research hoping to motivate researchers to join and contribute to the next work on the WAVE standards. In many cases, we identified the lessons learned from developing the current standards and defined why specific design choices have been made.

Lately, some cynical reviews of DSRC have been published in business magazines. A common set of arguments are shared by most skeptics. For one, the use of term "trial use" shaped the opinion that DSRC cannot be ready soon enough, and therefore, it is better to rely on alternative technology that can deliver now. A second argument is the lack of agreement on various technical issues like WAVE service layer and security. A third argument stems from common misunderstanding of the exact role DSRC plays and how various wireless technologies complement each other. Another argument questions the feasibility and the cost of deployment.

We maintain the view that DSRC is the base technology for future vehicular safety communications. Currently, DSRC is gaining popularity among researchers and most know technical issues are resolvable. Misconceptions about the technology will clear up as we promote DSRC in more and more technical and business gatherings. Parallel efforts on business, legal and legislative fronts will shape up the business models, address distractive concerns, and build up the case for gradual deployment.

Perhaps the most powerful support behind DSRC comes from two facts. First, the astute and well-crafted relation between IntelliDrive and DSRC which illustrate a situation where both IntelliDrive and DSRC fuel and support the success of each other while the failure of either initiative is 
not expected to drag the other one. The DSRC has been build with IntelliDrive in mind and therefore, offered services that are not available in WiMAX, WiFi, or cellular networks. There is currently a huge effort to integrate DSRC closely with Cellular services. An example would be the simTD project (http://www.simtd.de). Further discussion on DSRC integration with other technologies can be located in $[5,31]$. Another strong point in supporting DSRC is the availability of $75 \mathrm{MHz}$ bandwidth around the $5.9 \mathrm{GHz}$ dedicated for safety applications. In a conservative estimate, the DSRC bandwidth is evaluated to more than $\$ 20$ Billion. It would be irrational to shun this value because the system is not ready right now.

\section{Glossary of Terms}

AAT:

ACI:

AOIT:

ASTM:

ART:

CCF:

$\mathrm{CCH}:$

CCHI:

CICAS:

CRL:

CSMA/CA:

DSRC:

EDCA:

GPS:

I2V:

IETF: Internet Engineering Task Force Intelli-Drive : Vehicle-Infrastructure Integration IWBSS: Independent Basic Service Set

LoS: $\quad$ Line of Sight

MAC: $\quad$ Media Access Control

MEXT: $\quad$ Mobility EXTensions for IPv6

MLME: $\quad$ MAC Layer Management Entity

NEMO:

OBU:

PHY:

PKI:

PLME:

PSC:

PSID:

PST:

RFC:

RFID
RSU:

SAP:

SCH:

SCHI: Network Mobility

TSF: Timestamp Field On-Board Unit Physical Layer Public Key Infrastructure Physical Layer Management Entity Provider Service Context

Provider Service ID

Provider Service Table

Request for Comments

Radio Frequency Identification

Road-Side Unit

Service Access Point

Service Channel

Service Channel Interval
USID: User-Service-ID

UST: User Service Table

UTC: Coordinated Universal Time

V2V: Vehicle-Vehicle Communications

WAVE: Wireless Access in Vehicular Env.

WBSS: WAVE Basic Service Set

WME: WAVE Management Entity

WSA: WAVE Service Announcement

WSE: WAVE Security Entity

WSMP: WAVE Short-Message Protocol.

\section{References}

[1] National Center for Statistics and Analysis, "Traffic safety facts 2003," Report DoT HS 809 767, National Highway Traffic Safety Administration, U.S.-DoT, Washington, DC, USA, 2004.

[2] US Department of Transportation, "Vehicle Safety Communications Project," National Highway traffic Safety Admistration, Final Report, April, 2006.

[3] NHTSA, ITS Joint Program Office, "Report to Congress on the National Highway Traffic Safety Administration ITS Program, Program Progress During 1992-1996 and Strategic Plan for 1997-2002," Tech. Rep., U.S. Department of Transportation, Washington, DC, USA, 1997.

[4] T. Willke, P. Tientrakool, and N. Maxemchuk, "A survey of inter-vehicle communication protocols and their applications," IEEE Communications Surveys and Tutorials, vol. 11, no. 2, pp. 3-20, 2009.

[5] Y. Morgan, "Novel issues in DSRC vehicular communication radios," IEEE Canadian Review Magazine.

[6] J. G. Jordán, F. Soriano, D. Graullera, and G. Martín, "A comparison of different technologies for EFC and other ITS applications," in Proceedings of the IEEE Conference on Intelligent Transportation Systems (ITSC '01), pp. 1171-1176, Oakland, USA, August 2001.

[7] IEEE 802.11p SWG, et al., "Draft Amendment to Standard for Information Technology-Telecommunications and information exchange between systems-Local and Metropolitan networks_specific requirements-part 11: Wireless LAN Medium Access Control (MAC) and Physical Layer (PHY) specifications: Ammendment: Wireless Access in Vehicular Environments," IEEE 802.11p D6.0, June, 2009.

[8] IEEE P1609.3 SWG, et al., "Wireless Access in Vehicular Environments (WAVE) Networking Services," IEEE P1609.3 D1.2, June, 2009.

[9] IEEE P1609.4 SWG, et al., "IEEE P1609.4 Trial-use Standard for Wireless Access in Vehicular Environments (WAVE) Multi-Channel Operation,” IEEE P1609.4 D12, June, 2009.

[10] IEEE P1609.1 SWG, et al., "IEEE P1609.1 Trial-use Standard for Wireless Access in Vehicular Environments (WAVE) Resource Manager," IEEE P1609.1 D0.6, June, 2009.

[11] IEEE P1609.2 SWG, et al., "IEEE P1609.2 Trial-use Standard for Wireless Access in Vehicular Environments-Security Services for Applications and Menegement Messages," IEEE P1609.2 D07, June, 2009.

[12] J. Mar, et al., "A pilot sub-carrier-aided frequency equalizer for the dedicated short-range communications system over timevarying fading channels," in Proceedings of the Management and Technology Forum, China Communications, pp. 33-41, December 2006.

[13] J. Liu and J. Li, "Packet design and signal processing for OFDM-based mobile broadband wireless communication 
systems," IEEE Transactions on Mobile Computing, vol. 5, no. 9, pp. 1133-1142, 2006.

[14] T. Tsuboi, J. Yamada, N. Yamauchi, and M. Hayashi, "Dual receiver communication system for DSRC," in Proceedings of the 2nd International Conference on Future Generation Communication and Networking (FGCN'08), vol. 1, pp. 459464, Hainan Island, China, 2008.

[15] C. Cseh, "Architecture of the dedicated short-range communications (DSRC) protocol," in Proceedings of the 48th IEEE Vehicular Technology Conference (VTC '98), vol. 3, pp. 20952099, Ottawa, Canada, May 1998.

[16] International Radio Consultative Committee (CCIR), Report 517, Standard Frequency and Time-Signal Emissions: Detailed Instructions by SWG-7 for the Implementation of Recommendation 460 Concerning the Improved Coordinated Universal Time (UTC) System, January 1972, XIIth Plenary Assembly CCIR, New Delhi, India 1970, Geneva, International Telecommunication Union, 1970, Vol. III, 258a-258d; reprinted in Time and Frequency: Theory and Fundamentals, U.S. Monograph 140, Washington, DC, USA, U.S. Government Printing Office, 1974, 32-35. Reprinted by Geneva, International Telecommunication Union, Bureau, 1998.

[17] Y. Morgan and T. Kunz, "The full ESWAN destination-based approach; operations and evaluation," Journal of Information and Communications Technology, vol. 3, no. 2, 2007.

[18] Y. Morgan and T. Kunz, "PYLON-Lite: QoS model for gateways to ad-hoc network," Computers and Electrical Engineering, vol. 32, no. 1-3, pp. 68-87, 2005.

[19] H. T. Anh, J. Kim, W.-K. Cho, J. Choi, K. Lim, and J. Kwak, "A robust and low-complexity timing synchronization algorithm for ADSRC system," in Proceedings of the IFIP International Conference on Wireless and Optical Communications Networks WOCN '06, Tashkent, Uzbekistan, April 2006.

[20] S. Biswas, R. Tatchikou, and F. Dion, "Vehicle-to-vehicle wireless communication protocols for enhancing highway traffic safety," IEEE Communications Magazine, vol. 44, no. 1, pp. 74-82, 2006.

[21] M. Umemoto, "An experimental DSRC multimode terminal using software defined radio technology," in Proceedings of the IEEE Radio and Wireless Conference (RAWCON '01), pp. 165168, August 2001.

[22] A. Yokoyama and H. Harada, "Implementation of multichannel modem for DSRC system on signal processing platform for software defined radio," IEICE Transactions on Communications, vol. E89-B, no. 12, pp. 3225-3232, 2006.

[23] Y. Toor, P. Mühlethaler, A. Laouiti, and A. De La Fortelle, "Vehicle ad hoc networks: applications and related technical issues," IEEE Journal of Communications Survey and Turorials, vol. 10, no. 3, pp. 74-88, 2008.

[24] M. Fazio, C. E. Palazzi, S. Das, and M. Gerla, "Facilitating real-time applications in VANETs through fast address autoconfiguration," in Proceedings of the 4th Annual IEEE Consumer Communications and Networking Conference (CCNC '07), pp. 981-985, Las Vegas, Nev, USA, January 2007.

[25] M. Fazio, S. Das, C. E. Palazzi, and M. Gerla, "Automatic IP address configuration in VANETs," in Proceedings of the 3rd ACM International Workshop on Vehicular Ad Hoc Networks (VANET '06), MOBICOM '06, vol. 2006, pp. 100-101, Las Vegas, Nev, USA, 2006.

[26] G. G. Richard III, "Service advertisement and discovery: enabling universal device cooperation," IEEE Internet Computing, vol. 4, no. 5, pp. 18-26, 2000.

[27] I. Sedov, S. Preuß, C. Cap, M. Haase, and D. Timmermann, "Time and energy efficient service discovery in Bluetooth," in
IEEE Vehicular Technology Conference (VTC '03), vol. 57, no. 1, pp. 418-422, Jeju Island, Korea, April 2003.

[28] Z. Yue and L. Kwei-Jay, "Hierarchical management of service accountability in service oriented architectures," in Proceedings of the IEEE International Conference on Service-Oriented Computing and Applications (SOCA '07), pp. 55-62, June 2007.

[29] J. Yin, T. Elbatt, G. Yeung, et al., "Performance evaluation of safety applications over DSRC vehicular ad hoc networks," in Proceedings of the 1st ACM International Workshop on Vehicular Ad Hoc Networks (VANET '04), pp. 1-9, Philadelphia, Pa, USA, 2004.

[30] B.-S. Lee, C. S. Yim, D. H. Ahn, and D. G. Oh, "Performance evaluation of the physical layer of the DSRC operating in 5.8 $\mathrm{GHz}$ frequency band," ETRI Journal, vol. 23, no. 3, pp. 121$128,2001$.

[31] Y. Morgan, "Notes on DSRC and WAVE standards suite, its architecture, design, and characteristics," Journal of Computer Science and Technology, vol. 12, no. 4, 2010.
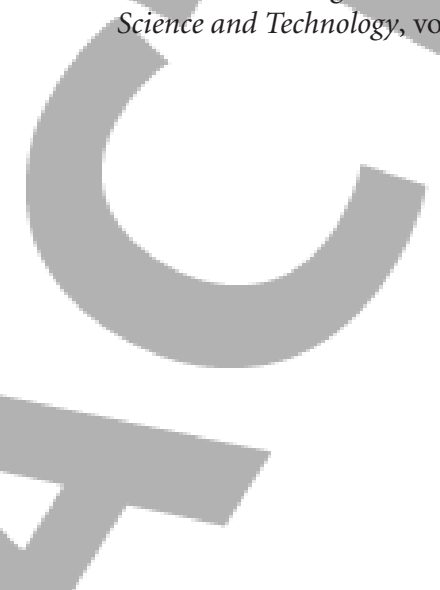